\title{
Distance versus energy fluctuations and electron transfer in single protein molecules
}

\author{
Jau Tang* \\ Center for Ultrafast Science and Technology, California Institute of Technology, Pasadena, California 91125, USA
}

Sheng-Hsien Lin

Institute of Atomic and Molecular Sciences, Academia Sinica, Taipei, Taiwan

(Received 15 March 2006; published 27 June 2006)

\begin{abstract}
Stochastic nature due to distance and energy fluctuations of single protein molecules involved in electrontransfer (ET) reactions is studied. Distance fluctuations have been assumed previously for causing the slow fluctuations in the ET rates between a donor-acceptor pair constrained to a native protein. Although the observed $t^{-1 / 2}$ power law can be derived using Langevin dynamics with a simple chain model, some discrepancies exist. The friction coefficient and the Rouse segment time constant deduced from experimental data are several orders of magnitude too large, even though the extracted force constant is reasonable. Therefore, questions are raised about the distance-fluctuation mechanism and the activationless ET hypothesis. As an alternative mechanism, we considered fluctuations in activation energy and analyzed the data from two different single protein experiments to determine spectral distribution of energy fluctuations.
\end{abstract}

DOI: 10.1103/PhysRevE.73.061108

\section{INTRODUCTION}

Understanding enzymatic and other biochemical reactions as well as conformational dynamics of biomolecules and polymers has been a subject of long-standing interest. Such information is often made more complex by a distribution among various conformations in ensemble-averaged studies. Developments over the past decade in techniques of singlemolecule fluorescence spectroscopy offer new tools to investigate events of physical, chemical, and biophysical importance $([1,2]$, and references cited therein). For example, chemical reactions and conformational dynamics of a single DNA or protein molecule can be studied in real time [1-6]. Many theoretical approaches to reaction kinetics modulated by conformational changes have been developed. Zwanzig [7] first analyzed first-passage time through a fluctuating geometric bottleneck. Subsequent studies on first-passage times through a similar bottleneck and other fluctuating barriers were considered by several authors including Wang and Wolynes [8], Szabo [9], Tachiya [10], and their co-workers. These first-passage-time studies assume normal Brownian diffusion, and lately Barkai et al. [11] considered, instead, a fractional Fokker-Planck equation for the anomalous diffusion problem.

Recently, other related approaches have been employed by Xie and co-workers [6,12-14] to improve the understanding of the stochastic nature of a single protein molecule. They beautifully demonstrated a single-molecule technique to probe protein conformational dynamics. By using a donoracceptor $(D-A)$ pair as a ruler, they measured the autocorrelation function $(\mathrm{ACF})$ of the fluctuations in the fluorescence lifetimes (inverse of the electron-transfer rate constant). In the single-molecule experiments, due to a time resolution of about $0.1 \mathrm{~ns}$, they observed fluorescence decays on the order of ns and some fluctuations in those decay rates. The time scale of those fluctuations occurs at a much slower time scale

*Corresponding author. Electronic address: jautang@caltech.edu
PACS number(s): 05.40.-a, 82.37.-j, 47.53.+n, 87.15.He

on the order of ms to $1000 \mathrm{~s}$. They attributed the causes to the induced distance fluctuations between the $D-A$ pair constrained to a protein. They reported an interesting $t^{-1 / 2}$ power-law temporal behavior in the distance ACF, and invoked fractional Brownian motion [14] to explain their data. Yang and Xie [15] considered reaction processes involving discrete states and Brownian diffusion processes.

More recently, there are some studies about the power-law temporal behavior of the distance ACF. Granek and Klafter [16] proposed an interesting model involving fraction, the vibrational excitation of a fractal, and showed that powerlaw dependence can be obtained. In a more recent study to account for the power law, Debnath et al. [17] considered a continuous-chain model. In another study, Tang and Marcus [18] independently considered a discrete-chain model. They all concluded that a chain model could reproduce the desired temporal behavior. The continuum model is a limit of the discrete Rouse chain model when the interchain spacing becomes very small, and both yield $t^{-1 / 2}$ power-law behavior. Although both models produce a qualitative feature of the power law similar to experimental data, there exist some qualitative discrepancies. As will be discussed later in this work, using a typical value for the friction coefficient a much shorter correlation time $(1-10 \mathrm{~ns})$ is obtained for the ACF, which is too short as compared to the reported value of $0.1-0.01 \mathrm{~s}[13,14]$. The extracted friction coefficient from their data according to the Rouse model is also many orders of magnitude too large as compared to those from other proteins and polymers by NMR and neutron scattering.

The organization of the materials in this paper is given in the following. After the Introduction in Sec. I, we will first examine the Rouse model in Sec. II, and analyze in Sec. II the experimental data. We will address several key problems associated with the distance-fluctuation model and show that chain dynamics is too fast to be responsible for the observed slow fluctuations in fluorescence-lifetime ACF. A more plausible alternative mechanism involving energy fluctuations is considered in Sec. IV. Finally, in Sec. V, some explanations are offered to address the question about whether the relevant ET is activationless or not, and also to reconcile the 
issue about ns fluorescence decays observed by Xie's group with the ps electron-transfer data from other groups. The hypothesis of activationless ET is also discussed.

\section{DISTANCE FLUCTUATIONS ACCORDING TO LANGEVIN DYNAMICS OF A ROUSE CHAIN}

In this section, we examine the distance-fluctuation mechanism for the ACF of fluorescence-lifetime fluctuations $[6,12-15,17,18]$. In these studies, the fluctuations in ET rates are assumed to be solely caused by conformationinduced distance fluctuations between a donor-acceptor pair constrained to a protein [6]. It is well known from the electron-transfer (ET) theory [19], that ET is given by the rate constant $\gamma=2 \pi\left|V_{e x}\right|^{2} \exp [-(\lambda$ $\left.\left.+\Delta G^{0}\right)^{2} / 4 \lambda k_{B} T\right] / \hbar \sqrt{4 \pi \lambda k_{B} T}$, where $\Delta G^{0}$ is the free energy gap, $\lambda$ the reorganization energy, and $\left|V_{e x}\right|^{2}$ the electronic coupling. They argued that the ET is near activationless $\left(\lambda \sim-\Delta G^{0}\right)$, and therefore the dominant factor for fluctuations in the rate is through the distance dependence in $V_{e x}$ $[6,13,14]$, where $\left|V_{e x}\right|^{2}$ decreases exponentially with the distance between a donor $(D)$ and an acceptor $(A)$. According to the distance-fluctuation model, conformational changes in a protein cause the $D-A$ distance and the ET rate to fluctuate in time, i.e., $\gamma(t)=k_{E T}^{0} \exp [-\beta \ell(t)]$, where $k_{E T}^{0}$ is the preexponential factor and $\ell(t)$ the fluctuating distance between the $D-A$ pair. The rate decreases exponentially with the $D$ - $A$ distance, with $\beta \sim 1.1-1.4 \AA^{-1}$ for proteins [20,21]. For activationless ET, fluctuations in ET rates are assumed to be caused mainly by distance changes.

The ACF of the lifetime fluctuations is defined as $\quad C_{2}(t) \equiv\left\langle\delta \gamma^{-1}(t) \delta \gamma^{-1}(0)\right\rangle /\left\langle\gamma^{-1}(t)\right\rangle\left\langle\gamma^{-1}(0)\right\rangle \quad$ [14]. With the definition of a quantity as $C_{2}(t) \equiv \exp \left[\beta^{2} C_{Q}(t)\right]-1$ and the property of white Gaussian random noise one can show that $\exp \left[\beta^{2} C_{Q}(t)\right]$ $=\langle\exp (\beta \ell(t)) \exp (\beta \ell(0))\rangle /\langle\exp (\beta \ell(t))\rangle\langle\exp (\beta \ell(0))\rangle[14]$ and $C_{Q}(t) \equiv\langle\delta \ell(t) \delta \ell(0)\rangle$, where $\delta \ell(t)=\ell(t)-\langle\ell(t)\rangle$ represents the deviation of the $D-A$ distance from its equilibrium separation.

With these assumptions, the results of the fluctuations in ET lifetimes provide information about the ACF of the distance fluctuations between the $D-A$ pair. In the studies of Xie and co-workers [6,12-15], ET is assumed to occur between a donor Tyr (tyrosine) and an acceptor FL (fluorescein) in the FL and anti-FL protein complex. This protein with a dimmer structure [22] illustrated in Fig. 1 and another protein of flavin:NADH oxidoreductase (not shown) are considered in the present study.

To investigate protein conformational dynamics and its effect on $C_{Q}(t)$, we assume that the donor and the acceptor are constrained to á chainlike protein structure. A Rouse chain [23-26] is a highly idealized model to mimic conformational dynamics of a protein shown in Fig. 2(a). A Rouse chain consists of identical elements, often termed Kuhn segments [24] which are schematically represented as beads shown in Fig. 2(b). Each segment usually contains a number of monomers, and is coupled to the nearest neighboring element by a harmonic force represented by a spring. In addition, in this simplified model the same harmonic force con-
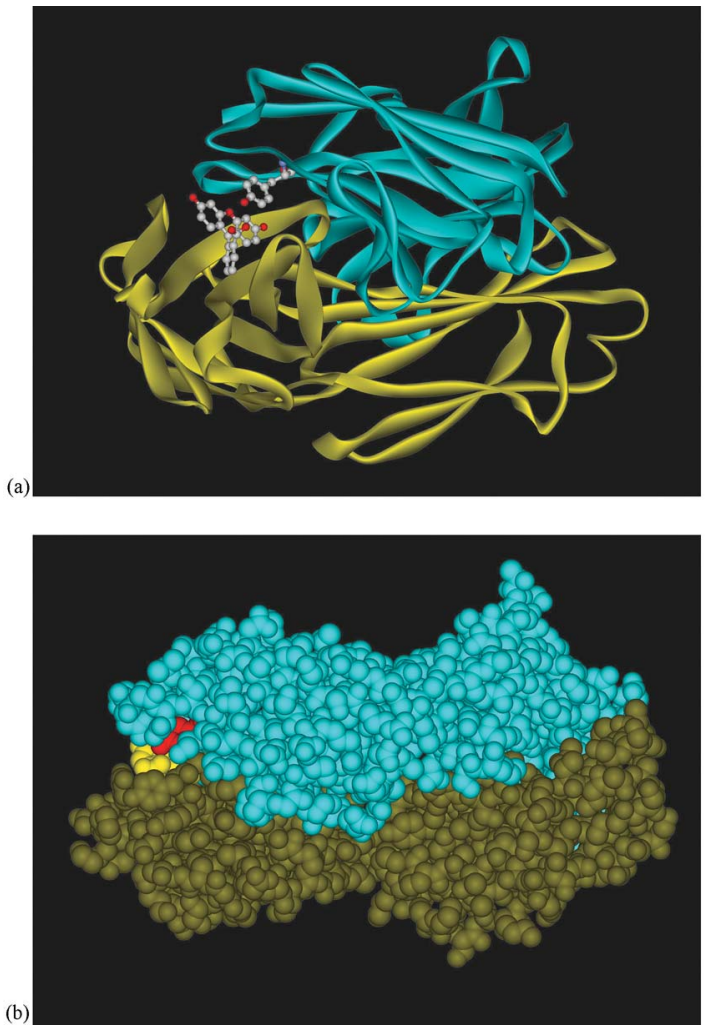

FIG. 1. (Color online) (a) Ribbon diagram of a FL and anti-FL protein, showing a dimeric structure with a donor Tyr (tyrosine) and an acceptor FL (fluorescein). (b) Ball-stick diagram of the same protein, showing that the native protein is highly compact and the donor-acceptor pair is located near the edge of the protein structure.

stant is assumed to couple the chain element to a donor or an acceptor. The Rouse model and other variations such as the Rouse-Zimm model have been applied to treat many structural and dynamic properties of polymers ([25], and references therein). They have also been used to model fluorescence-resonance energy transfer (FRET) in a single biopolymer [26], and other protein/DNA dynamics [27-30].

To describe the motion of a Rouse chain, one can use the multidimensional Langevin equation, often used in describing interacting particles under frictional forces [9]. We denote by $\mathbf{Q}$ as $\left(\mathbf{q}_{0}, \mathbf{q}_{1}, \mathbf{q}_{2}, \ldots \mathbf{q}_{\mathrm{N}-1}\right)$, where $\mathbf{q}_{\mathbf{k}}$ represents the displacement vector of a segment from its equilibrium position in an $N$-unit chain; the Langevin equation can be written as

$$
\begin{gathered}
\frac{d}{d t} \mathbf{q}_{\mathbf{0}}(t)+\frac{\omega^{2}}{\mathrm{~s}}\left[\mathbf{q}_{\mathbf{0}}(t)-\mathbf{q}_{\mathbf{1}}(t)\right]=F_{0}(t) / m \boldsymbol{s}, \\
\frac{d}{d t} \mathbf{q}_{\mathbf{k}}(t)+\frac{\omega^{2}}{\mathrm{~s}}\left[2 \mathbf{q}_{\mathbf{k}}(t)-\mathbf{q}_{\mathbf{k}-\mathbf{1}}(t)-\mathbf{q}_{\mathbf{k}+\mathbf{1}}(t)\right]=F_{\mathbf{k}}(t) / m \boldsymbol{\varsigma} \\
(k=1, N-2), \\
\frac{d}{d t} \mathbf{q}_{\mathbf{N}-\mathbf{1}}(t)+\frac{\omega^{2}}{\mathrm{~s}}\left[-\mathbf{q}_{\mathbf{N}-2}(t)+\mathbf{q}_{\mathbf{N}-\mathbf{1}}(t)\right]=F_{\mathbf{N}-1}(t) / m \boldsymbol{s},
\end{gathered}
$$

where $\zeta$ is the time-independent friction coefficient. Random force $\mathbf{F}(\mathrm{t})$ represents a memoryless, stationary Gaussian noise with zero mean and no correlation with the displacement of the oscillators in the chain. Nearest-neighbor 


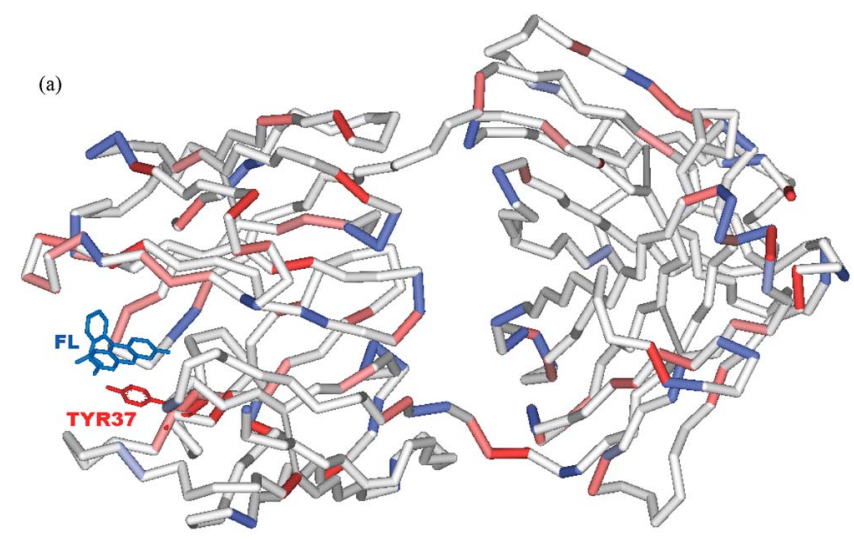

rium value $\left|\boldsymbol{q}_{n_{A}, e q}-\mathbf{q}_{n_{D} e q}\right|$. If the $D-A$ pair are attached to the ends of a chain, Eq. (2) reduces to

$C_{Q}(t)=\frac{8 k_{B} T}{m \omega^{2}} \sum_{n=o d d}^{N-1} \frac{1}{N} \cos ^{2}\left(\frac{n \pi}{2 N}\right) \exp \left[-\Gamma t \sin ^{2}\left(\frac{n \pi}{2 N}\right)\right]$,

which is valid for any chain length.

Assuming that a protein chain having a fixed force constant $m \omega^{2}$ and friction coefficient $\zeta$ is certainly an oversimplification for proteins. Instead of using a $\delta$-function distribution for $\Gamma$ at $4 m \omega^{2} / \zeta$, here we consider a variation to the Rouse model with a normalized distribution function $\vartheta(\Gamma)=\tau_{c}^{2} \Gamma \exp \left(-\Gamma \tau_{c}\right)$. With such an averaging, Eq. (3) becomes

$$
C_{Q}(t) / C_{Q}(0)=\frac{4}{N} \sum_{n=o d d}^{N-1} \frac{\cos ^{2}(n \pi / 2 N)}{1+\frac{t}{\tau_{c}} \sin ^{2}(n \pi / 2 N)} .
$$

In the limit of a large $N$, the above summation can be replaced by an integral [18], and yields

$$
\begin{gathered}
C_{Q}(t) / C_{Q}(0)=\frac{2}{1+\sqrt{1+t / \tau_{c}}}, \\
C_{Q}(0) \equiv 2 k_{B} T / m \omega_{c}^{2}, \quad \tau_{c} \equiv 1 / \Gamma_{c}, \quad \omega_{c}^{2} \equiv \Gamma_{c} \zeta / 4 .
\end{gathered}
$$

FIG. 2. (Color online) (a) Backbone structure (pKa plot) of the FL and anti-FL protein, showing the position of the donor Tyr and an acceptor FL. The edge-to-edge distance between the donor and the acceptor is $3.7 \AA$. (b) Schematic representation of a protein molecule by an ideal Rouse chain in 3D space, containing a donor $(D)$ and an acceptor $(A)$ with nearest neighbors coupled by a spring.

couplings are assumed by a harmonic potential with a force constant $m \omega^{2}$ [23].

Based on an estimate with $\beta \sim 1.1-1.4 \AA^{-1}$ for proteins, the ET rate is about $1 \mathrm{~ns}^{-1}$ for an activationless process. For electron transfer to occur within ns as observed in these proteins, the $D$ - $A$ distance cannot exceed $15 \AA$, i.e., the segment containing the donor and the segment containing the acceptor [Fig. 2(b)] must loop back within such a distance range, although the site-index difference $\left|n_{D}-n_{A}\right|$ could still be large. Assuming that the donor and the acceptor are attached to the chain at the bead index $n_{D}$ and $n_{A}$, according to our previous study [18], one can derive from the above Langevin equation the $\mathrm{ACF} C_{Q}(t)$ and is given by

$$
\begin{aligned}
C_{Q}(t)= & \langle\delta \ell(t) \delta \ell(0)\rangle \approx \frac{1}{3}\left\langle\left[\boldsymbol{q}_{\boldsymbol{n}_{\boldsymbol{A}}}(t)-\boldsymbol{q}_{\boldsymbol{n}_{\boldsymbol{D}}}(t)\right]\left[\boldsymbol{q}_{\boldsymbol{n}_{\boldsymbol{A}}}(0)-\boldsymbol{q}_{\boldsymbol{n}_{\boldsymbol{D}}}(0)\right]\right\rangle \\
= & C_{Q}(0) \sum_{n=1}^{N-1} \frac{1}{N}\left\{\cos \left[\frac{n \pi}{N}\left(n_{A}+\frac{1}{2}\right)\right]\right. \\
& \left.-\cos \left[\frac{n \pi}{N}\left(n_{D}+\frac{1}{2}\right)\right]\right\} \exp \left[-\Gamma t \sin ^{2}\left(\frac{n \pi}{2 N}\right)\right],
\end{aligned}
$$

where $\ell(t)=\left|\boldsymbol{q}_{\boldsymbol{n}_{A}}(t)+\boldsymbol{q}_{\boldsymbol{n}_{A}, e \boldsymbol{q}}-\boldsymbol{q}_{\boldsymbol{n}_{\boldsymbol{D}}}(t)-\mathbf{q}_{\mathbf{n}_{\mathrm{D}}, e \boldsymbol{q}}\right|$ is the distance for the $D-A$, and $\delta \ell(t)$ the distance deviation from its equilib-

Equation (5) shows an initial flat time dependence when the time $t$ is much shorter than $\tau_{c}$ and a subsequent $t^{-1 / 2}$ behavior. The time constant $\tau_{c}\left(1 / \Gamma_{c}\right)$ is related to the so-called segment relaxation time $\left(T_{s}=4 / \Gamma_{c} \pi^{2}\right)$ or the fundamental Rouse relaxation time by a constant [24]. At time $t$ much shorter than $\tau_{c}, C_{Q}(t)$ stays flat because perturbation at one end has not propagated to its next Kuhn segment. At longer times, the perturbation diffuses away like a one-dimensional (1D) diffusion to other segments along the chain, and $C_{Q}(t)$ decays according to $t^{-1 / 2}$ power law.

As the initial perturbation at one end propagates away and reaches the other end of a finite chain length, the power law breaks down as shown in Fig. 3 and $C_{Q}(t)$ decays as $\exp \left(-t / T_{D}\right)$ with a time constant $T_{R}=4 N^{2} / \Gamma \pi^{2}$, known as the Rouse relaxation time. In a DNA study of electrophoretic stretch and relaxation, Ferree and Blanch [31] showed a quadratic dependence of $T_{R}$ on $N$, in agreement with the Rouse model.

\section{ANALYSIS OF SINGLE-MOLECULE ET DATA BASED ON THE ROUSE MODEL}

In an analysis by Xie and co-workers [6,12-15], the ns fluorescence lifetime was measured to determine the ACF of the lifetime fluctuations. They assumed that the fluorescencedecay rate is dominated by an activationless ET and that the ACF reflects distance fluctuation between the $D-A$ pair. To fit $C_{Q}(t)$ a fractional Brownian noise (FBN) model [11] was applied by Kou et al. [13] and Min et al. [14] in terms of a Mittag-Leffler function with a free parameter $H(0<H<1)$. The experimental data were fitted well with $H=\frac{3}{4}$, whose microscopic origin remains to be determined. The present study is aimed at providing a step toward a better molecular 


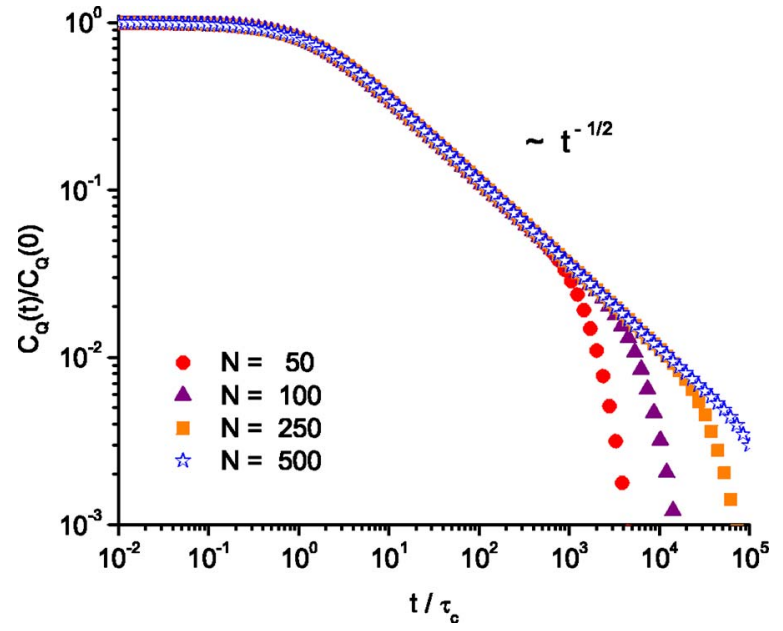

FIG. 3. (Color online) Distance ACF $C_{Q}(t) / C_{Q}(0)$ of Eq. (5) for various chain-segment units $N$ with a distributed $\Gamma$. At $t / \tau_{c} \ll 1$ when time $t$ is shorter than the correlation time $\tau_{c}, C_{Q}(t)$ remains flat and changes to $t^{-1 / 2}$ power law at $t / \tau_{c}>1$, and breaks down much later to decay single exponentially with a rate dictated by the chain length $N$

understanding of the origin of $H \sim \frac{3}{4}$ and the underlying mechanisms for the time dependence in $C_{Q}(t)$. With the approximations [6] that the quenching of fluorescence decay is dominated by ET and that the lifetime fluctuations directly reflect distance fluctuations in the ET rate constant, we next compare Eq. (5) with the experimental data.

As shown in Fig. 4(a), the observed data of Kou and Xie (taken from Fig. 2 of Ref. [13]), are fitted by the theoretical $C_{2}(t)$ for ET between the tyrosine donor (Tyr) and the flavin adenine dinucleotide acceptor (FAD) of the flavin:NADH oxidoreductase protein (Fre). The data of $C_{Q}(t)$ were converted from $C_{2}(t)$, and our fit is shown in Fig. 4(b) with a fitted $\beta^{2} C_{Q}(0)=0.32( \pm 0.01)$ and $\tau_{c}=0.007( \pm 0.001) \mathrm{s}$. With $\beta \sim 1.4 \AA^{-1}$, we have $C_{Q}(0)=0.16( \pm 0.01) \AA^{2}$. We have also compared the data and our fits with $C_{Q}(0) \exp \left(t / 4 \pi \tau_{c}\right) \operatorname{erfc}\left(\sqrt{t / 4 \pi \tau_{c}}\right)$. This functional form is equivalent to the Mittag-Leffler function [14] with $H=\frac{3}{4}$. We used the same value of $\tau_{c}$ obtained earlier by fitting to Eq. (5). In Fig. 5 we have also analyzed and fitted the data of Min et al. [14] for a different protein complex with the donor Tyr and the acceptor FL (protein structure shown in Fig. 1). We obtained a fitted value for $C_{Q}(0)=0.16( \pm 0.01) \AA^{2}$ and $\tau_{c}=0.11( \pm 0.01) \mathrm{s}$. In these two examples illustrated above, both Rouse and FBN models yield similar results within the bounds of experimental error, covering about six decades in time dependence.

With a fitted value of $\beta^{2} C_{Q}(0) \sim 0.32$ for both proteins, $C_{Q}(0) \sim 0.16 \AA^{2}$ if one uses $\beta \sim 1.4 \AA^{-1}$. Because $C_{Q}(t)=\langle\delta \ell(t) \delta \ell(0)\rangle$, we estimated the mean-square of distance fluctuations $\left\langle\delta \ell^{2}(0)\right\rangle$ to be about $0.16-0.26 \AA^{2}$. In a neutron scattering study of protein stiffness, Zaccai [32] obtained from purple membranes containing bacteriorhodopsin from $H$, salinaum an room-temperature value for $\left\langle\delta \ell^{2}(0)\right\rangle \sim 0.25 \AA^{2}$ and a force constant $\sim 2 \mathrm{~N} / \mathrm{m}$. In another neutron scattering study of CO-myoglobin [33],
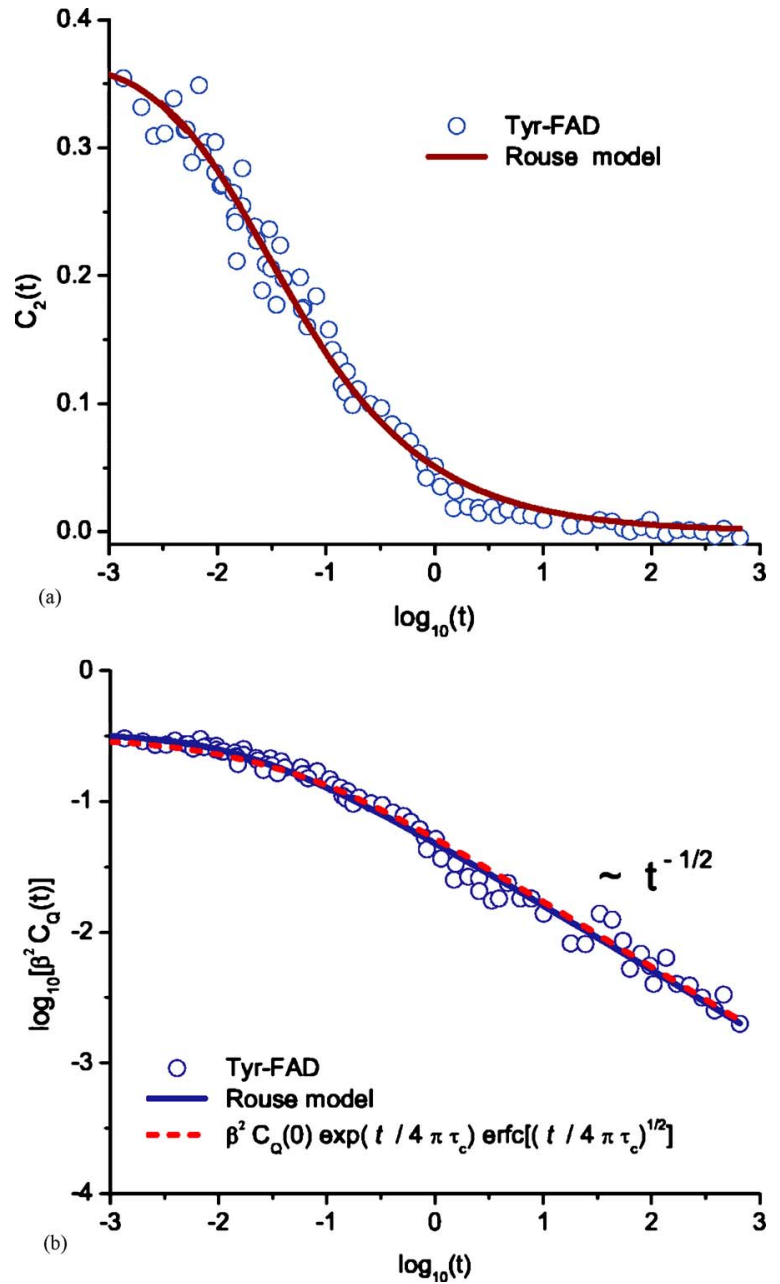

FIG. 4. (Color online) (a) ACF of fluorescence-lifetime fluctuations, $C_{2}(t)$, (solid curve), as compared to the room-temperature experimental data of Fre protein with a donor Tyr and an acceptor FAD. (b) Distance-fluctuation ACF $\beta_{B}^{2} C_{Q}(t)$ (solid curve) based on the distribution Rouse model of Eq. (5). The dash curve represents the Mittag-Leffler function with $H=3 / 4$ from the fractional Brownian noise (FBN) model. The time unit is $\mathrm{s}$.

$\left\langle\delta \ell^{2}(0)\right\rangle \sim 0.1 \AA^{2}$ and a force constant $\sim 1 \mathrm{~N} / \mathrm{m}$. From $C_{Q}(0) \sim 0.16 \AA^{2}$ and $k_{B} T \sim 25 \mathrm{meV}$, an effective force constant $m \omega_{c}^{2}$ between Kuhn segments is estimated to be about $320 \mathrm{meV}^{-2}$ or $5.1 \mathrm{~N} / \mathrm{m}$. The force constant for DNA using scanning polarization force microscopy [34] was found to be about $0.4 \mathrm{~N} / \mathrm{m}$. These values obtained by completely different techniques for other proteins are comparable to what we extracted from single-molecule ET data of Fre protein and FL-anti FL protein. In comparison with the force constant of a single $\mathrm{C}-\mathrm{C}$ bond of $440 \mathrm{~N} / \mathrm{m}$ [35], the effective coupling for the Rouse chain is two orders of magnitude smaller and is likely of the nature of van der Waals coupling. It indicates that the Rouse element-element restoring force is not strong enough to restrict some local flexibility. The extracted force constant in comparison with those of various biomolecules is given in Table I.

We compare next the friction coefficient $\zeta$ determined here with values from other techniques. In polymers a Kuhn segment of a few monomers is typical and $\zeta$ is on 
TABLE I. The fitted parameters for the effective Hook spring constant $m \omega_{c}^{2}$ according to the Rouse model.

\begin{tabular}{ccc}
\hline \hline & $m \omega_{c}^{2}(\mathrm{~N} / \mathrm{m})$ & Experiments (Refs.) \\
\hline Fre protein & 5 & Single-molecule ET [13] \\
FL-anti-FL & 5 & Single-molecule ET [14] \\
Protein & & Neutron scattering [32] \\
Purple membrane & 2 & Neutron scattering [33] \\
CO-Myoglobin & 1 & Scanning polarization \\
DNA & 0.4 & Force microscopy [34] \\
C-C single bond & 440 & {$[35]$} \\
\hline \hline
\end{tabular}

the order of $10 \mathrm{ps}^{-1}[22,36,37]$. According to $\tau_{c}=0.007$ and $0.11 \mathrm{~s}$, and $m \omega_{c}^{2}$ of $5 \mathrm{~N} / \mathrm{m}$ as extracted from Figs. 4(b) and 5 for two different proteins, with the effective mass for a Kuhn segment of about $50 \mathrm{amu}$, one estimates $\zeta$ to be on the order of $10^{24} \mathrm{~s}^{-1}$. Such a large $\zeta$ is apparently unphysical and is many orders of magnitude larger than a typical value of $10^{13} \mathrm{~s}^{-1}$ from myoglobin by time-resolved photoabsorption techniques [36] and from polymers [37] by NMR. In a NMR study of Rouse dynamics of a polyethylene oxide, Ries et al. [38] determined the fundamental Rouse relaxation time $T_{s}$ (or called segment relaxation time), or $4 \tau_{c} / \pi^{2}$, to be on the order of $1 \mathrm{~ns}$. By molecular-dynamics simulations using more realistic potentials than the simple harmonic potentials, Tsolou et al. [39] also obtained a similar nanosecond time constant for poly (butadiene). Arbe et al. [40] used neutron spin echos to determine the segment relaxation time of about $10 \mathrm{~ns}$. These ns time constants are seven to eight orders of magnitude faster than the values (0.01-0.1 s) inferred from Xie's data. The comparison of these values is listed in Table II. In proteins, there could be a range of distribution in $\zeta$. In dielectric relaxation measurements of several polymers at various temperatures a wide distribution in $\zeta$ is found [41]. Even with such a possible distribution, difficulties to settle the large discrepancy in $\tau_{c}$ or $\zeta$ remain.

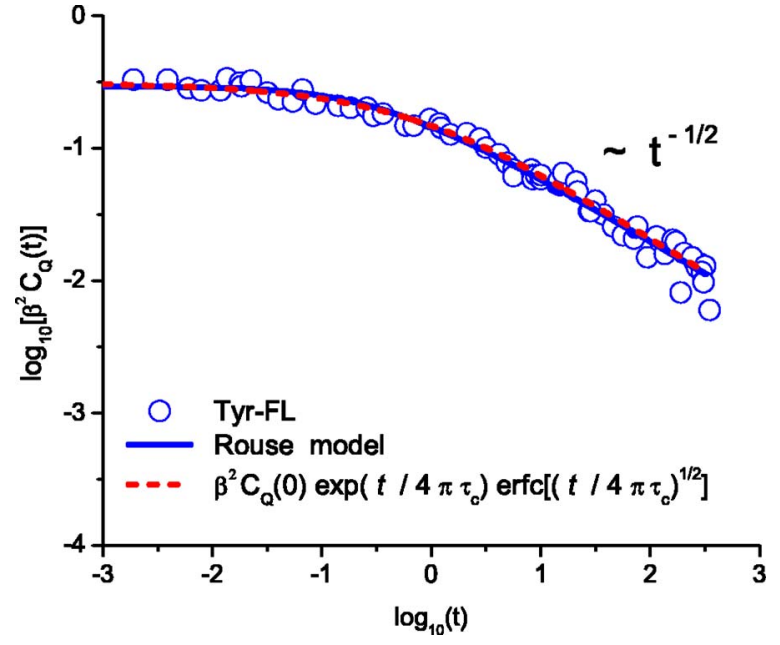

FIG. 5. (Color online) Distance-fluctuation ACF $\beta_{B}^{2} C_{Q}(t)$ (solid curve) from Eq. (5) and the FBN model vs the experimental data of FL and anti-FL complex with a donor Tyr and an acceptor FL. The time scale for $t$ is second.

In this analysis, we considered the case where the donor and the acceptor are attached to the ends of a chain. In the previous study [18], we derived a formula for $D-A$ at any position. If the $D-A$ pair were attached to other locations and with a large separation, the power law is not affected except that some small changes in $\tau_{c}$ arise [18]. Thus, the simplification used here is a valid approximate for the actual location of the $D-A$ pair in a protein structure. Our other study shows that if the $D-A$ pair is next to each other, the exponent of the power law could differ from $-\frac{1}{2}$, but that is not case for the protein systems considered here.

There are some difficulties for the Rouse model as an explanation to Xie's experimental data. First of all, with or without a distribution in $\Gamma$ the value of $\tau_{c}$ is affected by less than one order of magnitude. If one were to accept the ordinary value for the friction coefficient on the order of $10^{-12} \mathrm{ps}^{-1}$, then the deduced value for the correlation time $\tau_{c}$ should be on the order of 1-10 ns. Therefore, one may

TABLE II. The fitted parameters for the segment relaxation time $\tau_{c}$, the coupling frequency $\omega_{c}$, and the friction coefficient $\zeta$ according to the Rouse model. The effective mass $m$ for a Kuhn segment is set at $50 \mathrm{amu}$ as an approximation, assuming a segment of 4-5 monomers as often used for polymers [24]. Here $\omega_{c}^{2}=\zeta / 4 \tau_{c}, \tau_{c}$ and $\omega_{c}$ are defined in Eq. (5).

\begin{tabular}{ccccc}
\hline \hline & $\begin{array}{c}\tau_{c} \\
(\mathrm{~s})\end{array}$ & $\begin{array}{c}\omega_{c} \\
\left(\mathrm{ps}^{-1}\right)\end{array}$ & $\begin{array}{c}\zeta \\
\left(\mathrm{ps}^{-1}\right)\end{array}$ & Experiments (Refs.) \\
\hline $\begin{array}{c}\text { Fre protein } \\
\begin{array}{c}\text { FL-anti-FL } \\
\text { protein }\end{array}\end{array}$ & 0.007 & 7.7 & $1.7 \times 10^{12}$ & $\begin{array}{c}\text { Single-molecule ET [13] } \\
\text { Single-molecule ET [14] }\end{array}$ \\
$\begin{array}{c}\text { Fe-myoglobin } \\
\text { Poly (butadiene) }\end{array}$ & N.A. & 3.9 & $2.6 \times 10^{13}$ & Optical absorption [36] \\
$\begin{array}{c}\text { Poly } \\
\text { (ethylene oxside) }\end{array}$ & N.A. & N.A. & 60 & NMR [37] \\
$\begin{array}{c}\text { Poly } \\
\text { (vinyl ethylene) }\end{array}$ & $10^{-9}$ & N.A. & N.A. & NMR [38] \\
Poly (butadiene) & $10^{-9}$ & N.A. & N.A. & Neutron spin echo [39] \\
\hline \hline
\end{tabular}




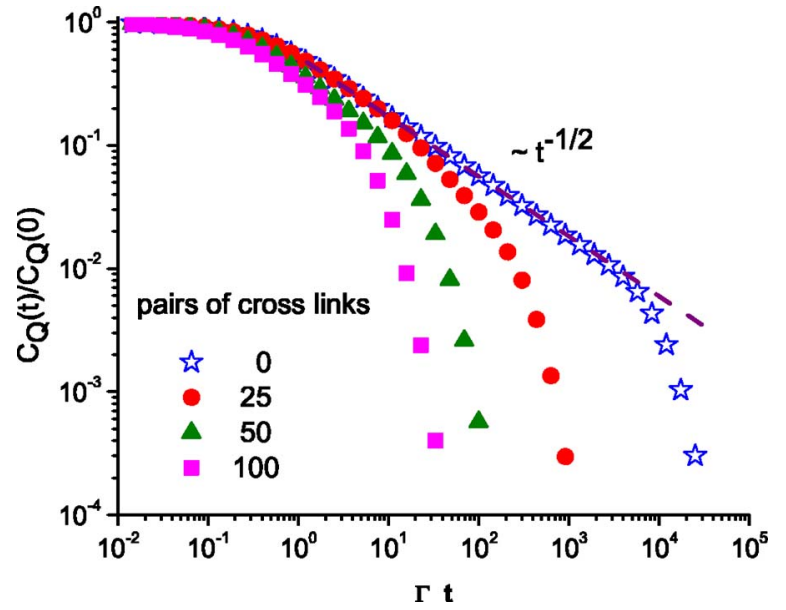

FIG. 6. (Color online) Distance-fluctuation $\mathrm{ACF} C_{Q}(t) / C_{Q}(0)$ versus a number of pairs of cross links among beads other than the nearest neighbor couplings. A chain of 250 units is considered with an ensemble average over randomly chosen cross links. The presence of cross links could severely damage the simple $t^{-1 / 2}$ powerlaw behavior of an ideal Rouse chain.

wonder why the correlation time $\tau_{c}$, or the segment relaxation time $T_{s}\left(T_{s}=4 \tau_{c} / \pi^{2}\right)$, is so long in Xie's $C_{2}(t)$ data. Second, for FL-anti FL protein, there are about $250 \mathrm{C}-\mathrm{C}$ or $\mathrm{C}-\mathrm{N}$ bonds along the backbone, so the Rouse segment number $\mathrm{N}$ cannot exceed 250, and is much smaller if one takes five monomers per a Rouse segment in usual polymers [24]. With $\tau_{c}$ of $0.007 \mathrm{~s}$ for Fre protein and $0.11 \mathrm{~s}$ for FL-anti-FL protein and $N$ of 50, according to Fig. 3, the exponential tail should become noticeable around 20 and $300 \mathrm{~s}$, respectively. However, such a long-time exponential tail is not present in the observed single protein data [Figs. 4(b) and 5].

In addition, there could be many possible cross links for a native protein which appears to be more like a folded twine rather than a stretched out string, as illustrated Fig. 1(b). These additional links would make the interbead coupling look more like a 3D coupling network than a $1 \mathrm{D}$ network. The cross-link couplings, for those beads coming within $10 \AA$ range of van der Waals interaction, could cause significant deviations from the ideal $t^{-1 / 2}$ power law. As illustrated in Fig. 6, as the number of the cross-linked pair increases, the power law starts to give way to a more dominant exponential tail.
In the distance-fluctuation model, the ET was assumed to be activationless so that the changes in the rate arise solely from fluctuations in the electronic coupling through its dependence on the $D-A$ distance. However, because the $D-A$ has a separation $\ell$ of about $4 \AA$, if the ET was activationless, one estimates the pre-exponential factor $k_{E T}^{0}=2 \pi e^{-\beta \ell} / \hbar \sqrt{4 \pi \lambda k_{B} T}$ to be on the order of $\mathrm{ps}^{-1}$ for $\beta \sim 1.1-1.4 \AA^{-1}$ for proteins [20,21], instead of $\mathrm{ns}^{-1}$ as observed in Xie's experiments.

In short, there are four major unsettled issues that compel us to question the validity of the distance-fluctuation model, i.e., (1) the extracted friction coefficient $\zeta$ and $\tau_{c}$ appear too large; (2) the long-time exponential tail should occur but has not been observed; (3) deviations from the simple power law by cross links in native proteins could be severe; (4) ET might not be activationless in those proteins considered here. More details will be given in Sec. V.

\section{ENERGY-FLUCTUATION MODEL AS AN ALTERNATIVE}

In the above section, we have discussed some difficulties associated with the distance-fluctuation hypothesis and Rouse chain dynamics. Here we will consider a different mechanism as a more plausible alternative. Other than the distance-induced fluctuations, slow fluctuations in the activation energy could also cause electron-transfer rates to fluctuate. The electron-transfer rate $\quad \gamma=2 \pi\left|V_{e x}\right|^{2} \exp \left(-\beta_{B} E_{A}\right) / \hbar \sqrt{4 \pi \lambda k_{B} T}$ contains a Boltzmann factor $\exp \left(-\beta_{B} E_{A}\right)$. If the activation energy $E_{A}(t)$ fluctuates in time due to some very slow conformational changes (ms or longer) in the proteins, so does the rate $\gamma(t)$. Here we model $Q(t)$, defined by $E_{A}(t)-E_{A, e q}$, as a sum of stochastic variables $q_{k}(t)$ that represents the displacement of an oscillator, with $Q(t)=\Sigma_{k} g_{k} q_{k}(t)$ and $H_{B}=\Sigma_{k} H_{k}=\Sigma_{k} p_{k}^{2} / 2 m_{k}+m_{k} \omega_{k}^{2} q_{k}^{2} / 2$. One has

$$
\begin{aligned}
\left\langle\gamma^{-1}(t) \gamma^{-1}(0)\right\rangle & =\gamma_{e q}^{-2}\left\langle e^{\beta_{B} Q(t)} e^{\beta_{B} Q(0)}\right\rangle \\
& =\frac{\operatorname{Tr}_{B}\left(e^{-\beta_{B} H_{B}} e^{\beta_{B} Q(t)} e^{\beta_{B} Q(0)}\right)}{\operatorname{Tr}_{B}\left(e^{-\beta_{B} H_{B}}\right)},
\end{aligned}
$$

for the $k$ th oscillator. The trace involving noncommuting operators $e^{\beta_{B} Q(t)}$ and $e^{\beta_{B} Q(0)}$ can be calculated and is given by [42]

$$
\operatorname{Tr}_{B}\left(e^{-\beta_{B} H_{k}} e^{\beta_{B} q_{k}(t)} e^{\beta_{B} q_{k}(0)}\right)=\frac{\exp \left\{\frac{\beta_{B}^{2}}{2 m_{k}} \frac{\hbar g_{k}^{2}}{\omega_{k}}\left[\operatorname{coth}\left(\frac{\beta_{B} \hbar \omega_{k}}{2}\right)\left[1+\cos \left(\omega_{k} t\right)\right]-i \sin \left(\omega_{k} t\right)\right]\right\}}{2 \sinh \left(\frac{\beta_{B} \hbar \omega_{k}}{2}\right)}
$$

The above relation is related to the Huang-Ryes factor [43] widely used in the literature of electron and/or energy transfer. By summation over all oscillators one obtains from Eq. (6)

$$
\left\langle\delta \gamma^{-1}(t) \delta \gamma^{-1}(0)\right\rangle=\left\langle\gamma^{-1}(0)\right\rangle^{2}\left[e^{\beta_{B}^{2} C_{Q}^{(t)}}-1\right]
$$


where

$$
\begin{gathered}
C_{Q}(t)=\sum_{k} \frac{\hbar g_{k}^{2}}{2 m_{k} \omega_{k}} \operatorname{coth}\left(\frac{\beta_{B} \hbar \omega_{k}}{2}\right) \cos \left(\omega_{k} t\right), \\
\left\langle\gamma^{-1}(0)\right\rangle^{2}=\gamma_{e q}^{-2} \exp \left[\beta_{B}^{2} \sum_{k} \frac{\hbar g_{k}^{2}}{2 m_{k} \omega_{k}} \operatorname{coth}\left(\frac{\beta_{B} \hbar \omega_{k}}{2}\right)\right],
\end{gathered}
$$

and the normalized autocorrelation function $C_{2}(t)$ is given by $C_{2}(t) \equiv \exp \left[\beta_{B}^{2} C_{Q}(t)\right]-1$. One can define the spectral density of the bath oscillators as $J(\omega) \equiv \Sigma_{k}\left(g_{k}^{2} / m_{k} \omega_{k}^{2}\right) \delta\left(\omega-\omega_{k}\right)$. At the high temperature limit, one has

$$
C_{Q}(t) \approx \beta_{B}^{-1} \sum_{k} \frac{g_{k}^{2}}{m_{k} \omega_{k}^{2}} \cos \left(\omega_{k} t\right)=\beta_{B}^{-1} \int_{0}^{\infty} d \omega \cos (\omega t) J(\omega) .
$$

The temporal behavior of $C_{Q}(t)$ depends on the details of the spectral distribution of the bath modes. Here we first consider the following spectral density $J(\omega)$ :

$$
J(\omega)=\frac{J_{0}}{\Omega_{c} \sqrt{\pi}}\left(\frac{\omega}{\Omega_{c}}\right)^{-1 / 2} \exp \left(-\omega / \Omega_{c}\right),
$$

where $\Omega_{c}$ represents a characteristic frequency for the exponential drop off. The choice of such a $J(\omega)$ allows us to derive a simple result from Eq. (10) as

$$
C_{Q}(t) / C_{Q}(0)=\frac{1}{\sqrt{2}}\left(1+\Omega_{c}^{2} t^{2}\right)^{-1 / 4} \sqrt{1+\frac{1}{\sqrt{1+\Omega_{c}^{2} t^{2}}}},
$$

where $C_{Q}(0)=\beta_{B}^{-1} J_{0}$. $C_{Q}(t) / C_{Q}(0)$ is close to 1 at short times and becomes $t^{-1 / 2}$ at longer times, a similar behavior observed by Kou and Xie [13] and Min et al. [14].

The temporal behavior at $t \sim 1 / \Omega_{c}$, i.e., around the bending regime between the initial flat plateau and the subsequent power-law decay, depends on the details of $J(\omega)$ around $\Omega_{c}$. Here we consider a more general spectral density $J(\omega)$ with a stretched exponential dependence as

$$
J(\omega)=\frac{J_{0} \alpha}{\Omega_{c} \Gamma[(\xi+1) / \alpha]}\left(\frac{\omega}{\Omega_{c}}\right)^{\xi} \exp \left[-\left(\frac{\omega}{\Omega_{c}}\right)^{\alpha}\right],
$$

and $\Gamma$ is the gamma function as a normalization constant, the choice of the parameters $\xi$ and $\alpha$ needs to maintain finite integral for the overall spectral distribution. If $\alpha=1$ the ACF in Eq. (10) for the above $J(\omega)$ can be obtained in a simple analytic form as

$$
C_{Q}(t) / C_{Q}(0)=\left(1+\Omega_{c}^{2} t^{2}\right)^{-(1+\xi) / 2} \cos \left[(1+\xi) \tan ^{-1}\left(\Omega_{c} t\right)\right],
$$

which reduces to Eq. (12) if $\xi=-\frac{1}{2}$. Equation (14) shows that $C_{Q}(t)$ remains constant if $t$ is much shorter than $1 / \Omega_{c}$ but changes later to a power law of $t^{-(1+\xi)}$ at a longer time.

Generally speaking, one can reproduce the desired temporal behavior so long as the spectral density $J(\omega)$ has a $\omega^{-1 / 2}$ dependence and a rapid cutoff at $\Omega_{c}$. Taking Laplace transforms of Eq. (10), for any $J(\omega)$ one has

$$
\bar{C}_{Q}(s)=\beta_{B}^{-1} \int_{0}^{\infty} d \omega \frac{s}{s^{2}+\omega^{2}} J(\omega)=\beta_{B}^{-1} \int_{0}^{\infty} d z \frac{J(s z)}{1+z^{2}} .
$$

The above integral has a major contribution at a small $z$. If $J(s z)$ behaves as $(s z)^{\xi}$ at small $s z$, then $\bar{C}_{Q}(s)$ behaves as $s^{\xi}$, i.e., $C_{Q}(t)$ is proportional to $t^{-(1+\xi)}$. At a much shorter time, $C_{Q}(t)$ remains constant. Because of the observed log time $t^{-1 / 2}$ power law, one can conclude that $\xi=-\frac{1}{2}$. The factor $\alpha$ controls the bending and needs to be determined by actual data fitting.

According to this energy-fluctuation model, we have fitted both data sets for different proteins, which were analyzed previously in Figs. 4(b) and 5 by the Rouse model. Numerical integration in Eq. (10) was computed using $J(\omega)$ of Eq. (13). The fitted curve and the raw data are illustrated in Fig. 7 , covering six decades of time dependence, and the fit is within the bounds of experimental error. It is found that if $\alpha \sim \frac{1}{2}$ and $\xi \sim-\frac{1}{2}$, i.e.,

$$
J(\omega)=\frac{J_{0}}{2 \Omega_{c}} \sqrt{\frac{\Omega_{c}}{\omega}} \exp \left(-\sqrt{\frac{\omega}{\Omega_{c}}}\right),
$$

the desired temporal behavior can be reproduced. From the fits to both data sets, it is estimated that $J_{0} \sim 8 \mathrm{meV}$ and $\Omega_{c} \sim 16 \mathrm{~s}^{-1}$ for Fre protein with a Tyr-FAD in Fig. 7(a) and $\Omega_{c} \sim 1 \mathrm{~s}^{-1}$ for FL and anti-FL complex with Try-FL in Fig. $7(\mathrm{~b})$.

The observed long-time $t^{-1 / 2}$ behavior from the experiments might indicate that the spectral distribution $J(\omega)$ for the activation-energy fluctuations due to very slow protein conformational dynamics could follow an asymptotic $\omega^{-1 / 2}$ dependence. In fluorescence intermittency of quantum dots, the fluctuation in energy space as a 1D random-walk process is considered as the underlying mechanism, and the effects on diffusion-controlled reaction dynamics are attributed to the cause for the power-law blinking statistics [44]. Similarly, slow energy fluctuations considered here for an activated electron-transfer process might play a role for the $t^{-1 / 2}$ power law in the fluorescence-lifetime fluctuations of Xie's single protein molecule experiments. A molecular-based model for such slow energy fluctuations and their actual spectral distribution await further studies.

\section{DISCUSSION AND CONCLUSIONS}

In summary, to explain the observed power-law behavior in the ACF of lifetime fluctuations in the single protein experiments, we have considered and compared two possible mechanisms, i.e., the distance-fluctuation model due to chain dynamics and the newly proposed alternative assuming energy fluctuations. The power-law behavior for distance vs energy fluctuations, and their relation to fluctuating fluorescence lifetimes in single-molecule ET experiments were analyzed. Comparisons of the extracted parameters with the values of other proteins and polymers by different methods were also made.

In this paper, we first considered a Rouse model and Langevin dynamics to mimic distance fluctuations between the $D-A$ pair in a chain. We analyzed existing experimental 

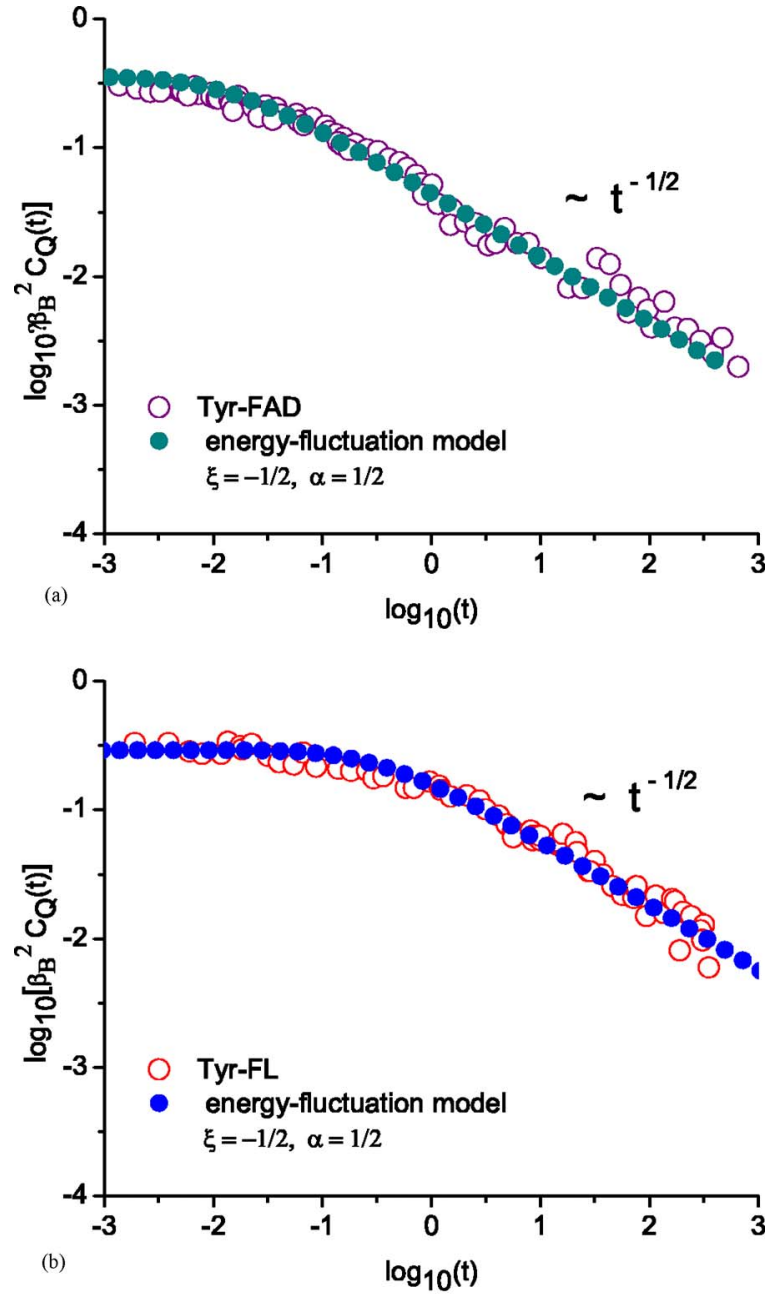

FIG. 7. (Color online) (a) Energy-fluctuation $\mathrm{ACF} \beta_{B}^{2} C_{Q}(t)$ of fluorescence-lifetime fluctuations data of Fre protein with a TyrFAD pair. (b) Energy-fluctuation $\mathrm{ACF} \beta_{B}^{2} C_{Q}(t)$ for FL and anti-FL complexes with Tyr-FL pair. Data were fitted by Eq. (10) of the energy-fluctuation model using the spectral density function of Eq. (16), i.e., a special case with $\alpha \sim \frac{1}{2}$ and $\xi=-\frac{1}{2}$ for Eq. (13). It is found that $J_{0} \sim 8 \mathrm{meV}$ for both fits and $\Omega_{c} \sim 16 \mathrm{~s}^{-1}$ for Fig. 7(a) and $\Omega_{c} \sim 1 \mathrm{~s}^{-1}$ for Fig. 7(b). The unit for $t$ is $\mathrm{s}$.

data sets for two different proteins (Figs. 4 and 5) by Xie's group, and determined the effective force constant $m \omega_{c}^{2}$ to be about $5 \mathrm{~N} / \mathrm{m}$ (Table I), which is comparable to other proteins from neutron scattering measurements. However, the extracted value for the correlation time $\tau_{c}$ for the distance ACF and the friction coefficient $\zeta$ (Table II) are many orders of magnitude too large than those of myoglobin and polymers by other techniques. If a reasonable value for $\omega_{c}$ and $\zeta$ (Table II) was used, which is on the similar order of magnitude for both proteins and polymers, the fluctuations in ET rates due to chain dynamics would occur at a much shorter $\tau_{c}$ on the order of 1-10 ns, instead of $0.01-0.1 \mathrm{~s}$ from the reports of Xie's group [13,14].

The Rouse chain model of Sec. II, which we considered as an implausible mechanism, also yields a long-time $t^{-1 / 2}$ power law in ACF. The presence of such a power law is not surprising. Equation (1) for a chain with nearest-neighbor couplings is mathematically equivalent to a random-walk process along 1D discrete sites [45] with $\omega^{2} / \mathrm{s}$ as the hopping rate between sites. In our recent different study, we have also shown that if the $D$-A distance becomes very small, the ordinary power dependence of $t^{-1 / 2}$ for $D-A$ at a large separation could change into $t^{-31 / 2}$ and $t^{-5 / 2}$, depending on the topological positions of the $D-A$ pair [46]. Although the distance-fluctuation model with the Rouse chain dynamics can produce $t^{-1 / 2}$ dependence for the ACF, the time scale is off by too many orders of magnitude. It cannot be solely responsible for the observed power-law behavior in the time scale of seconds. Such a large discrepancy cannot be reconciled even if one replaces the discrete chain model by a continuous model [17] or other modified Rouse models such as the Rouse-Zimm model $[25,26]$.

In addition, for these proteins with about $250 \mathrm{C}-\mathrm{C}$ or $\mathrm{C}-\mathrm{N}$ units, the Rouse segment number $N$ is about 50, and for Fre protein with $\tau_{c}$ of $0.007 \mathrm{~s}$ a long-time exponential tail is expected to appear beyond $20 \mathrm{~s}$. However, such a tail has not been observed. Moreover, these native folded proteins could contain numerous cross links and the exponential tail would occur even sooner. Although we believe that the chain dynamics and induced distance fluctuations between the $D-A$ pair could cause the electron-transfer rate to fluctuate in time but the effects should occur at a much short time scale $(\sim \mathrm{ns})$. With a bin time on the order of $\mathrm{ms}$ in the singlemolecule experiments [6], the rapid distance-fluctuation effects would not be detectable. The observed slow fluctuations in fluorescence decays could arise due to a different mechanism such as energy fluctuations suggested in Sec. IV.

Other than the above difficulties that the distancefluctuation model with a Rouse chain has to face, such a model also relies on the assumption that the ET is activationless so that the fluctuating ET rate is solely due to distance changes. An activationless ET on the order ns is too slow for a $D-A$ pair separated by only $4 \AA$. In a femtosecond dynamics study of flavoproteins, Zhong and Zewail [47] observed nonexponential fluorescence decay with dominant fast decaying components on the order of $1-50$ ps and a very small component with a much slower decay at ns. The origin of the ns components is presently not clear. With a $D-A$ separation of about $4 \AA$ [Fig. 2(a)], if ET were near activationless as previously assumed the decay should occur on the time scale of ps rather than ns. There are some possible causes for the ns component. The acceptor FL may not be in its usual position inferred from the X-ray crystal structure or the hypothesis of activationless ET for such a component might not be valid. The observed ns components might be due to a delayed fluorescence phenomenon, arisen from a combined process involving a fast forward electron transfer from the photoexcited state $(D A)^{*}$ to a charge-separated state $D^{+} A^{-}$ and a much slower reverse electron transfer back to the photoexcited state. The resultant fluorescence decay from the photoexcited state to the ground state, according to such a three-level scheme, would result in a nonexponential decay with a dominant fast (ps) decaying component and a smaller slowly (ns) decaying component. By setting up and solving the coupled rate equations for such a three-state scheme involving $(D A)^{*}, D^{+} A^{-}$, and the ground state, one can show that the fluorescence intensity which depends on the population in $(D A)^{*}$ decays nonexponentially as a result of the re- 
verse ET from $D^{+} A^{-}$back to $(D A)^{*}$. Although the forward charge separation from $(D A)^{*}$ to $D^{+} A^{-}$can be near activationless and very fast, the reverse ET process could be activated and much slower. Therefore, the observed slow fluctuations in the ns component of electron-transfer processes might not be caused by fluctuations in the $D-A$ distance modulated by chain dynamics. The ns decaying component might represent delayed fluorescence decay as a result of the much slower reverse ET which is an activated process and the fluctuations in the ET rate might well be caused by fluctuations in the activation energy due to very slow conformational changes of the proteins.

The energy-fluctuation model offered in Sec. IV might provide a more plausible explanation to the origin of the observed power law of $t^{-1 / 2}$ than the distance fluctuations of the Rouse chain. The harmonic model used here for fluctuating energy is similar to the spin-boson model [42] used in the electron-transfer theory. The energy-fluctuation model discussed here involves a very slow motion of proteins in a time scale of ms or longer. These slow modes are different from the very fast solvent dynamics (subpicosecond or longer) that facilitates the electron-transfer reactions. The extremely slow motion inferred from Xie's experiments indicates that the motion might involve a large inertial, i.e., a much greater portion of the protein structure near the $D-A$ pair.

The fitted specific spectral distribution obtained here could provide a guideline for the search for a better molecular-based mechanism as an alternative to the Rouse chain model (and its many variations) considered previously by us and others. An interesting remark needs to be pointed out that the frequency dependence in Eq. (16) resembles the Green function in the Laplace-transform $s$ domain for a 1D diffusion equation, i.e., $\sqrt{1 / 4 D s} \exp \left(-\sqrt{s \Delta E^{2} / D}\right)$. The $\omega^{-1 / 2}$ dependence in Eq. (16) (or $s^{-1 / 2}$ here) as the best-fitted spectra distribution might relate to a $1 \mathrm{D}$ random-walk process in energy space. The cutoff frequency $\Omega_{c}$ (or $D / \Delta E^{2}$ here) in the exponential factor could represent the reciprocal time for the diffusion in energy to reach $\Delta E$, a characteristic energy scale, possibly related to the energy difference between a starting and an ending states. It may be related to a $1 \mathrm{D}$ diffusion involving a double-well potential or some gated 1D diffusion processes. Details about such a possible connection and model calculations await further investigations.

The fractional Brownian noise (FBN) model [11] employed by Xie and co-workers $[13,14]$ was previously assumed for a random distance variable in the distancefluctuation scheme. It could be recast as a random energy variable in the present energy-fluctuation scheme. The physical origin for the Mittag-Leffler parameter $H=\frac{3}{4}$ remains to be explored and better understood on a molecular level. As an effort to interpret the temporal behavior of the fluorescence-lifetime ACF, Min and Xie [48] recently studied Kramers' reaction in a double-well potential. They used a generalized Langevin equation assuming a specific powerlaw friction kernel. It would be interesting to show the origin for the specific power for the power-law kernel, which is not clear presently. Conformational changes and energy fluctuations in proteins are better characterized by Kramers' reaction in a double-well potential with a flat top. Such kind of reaction is different from Marcus' ET reaction that involves two parabolas with a cusplike energy-level crossing. It would be interesting to see if the extension could lead to the desired power-law behavior in the ACF without invoking nonMarkovian processes with an ad hoc power power-law friction kernel. A non-Markovian process could arise from a Markovian process but with dynamics involving internal degrees of freedom. For example, Langevin equations for particles constrained to a chain (coupled Markovian equations) lead naturally to a power law which could also be obtained by a generalized Langevin equation (non-Markovian equation) for a single particle, i.e., a retarded friction kernel could be caused by some intrinsic dynamics. By identifying the internal degree of freedom and incorporating it to a 1D diffusion, such as incorporating Kramers' double-well potential in a Markovian-type 1D diffusion-controlled reaction [44], one might be able to obtain results equivalent to nonMarkovian equation with a retarded friction kernel. The development of these ideas on such an extension deserves further studies.

Electron transfer in single protein molecules involves complex environmental changes and a multidimensional description of energy landscape may be needed $[49,50]$. Our model for slow energy fluctuations provides a simple description to account for the power-law behavior and its relationship to the spectral distribution of fluctuations. A recent molecular-dynamics (MD) study by Kneller and Hinsen [51,52] indicates a power-law distribution in the $\mathrm{GHz}$ scale for lysozyine. The protein dynamics at a much longer time scale ( $\mathrm{ms}$ to $\mathrm{s}$ ) relevant to the present study could have different behavior and is presently unknown. Extending MD calculations to such a long time scale is expensive, and a better numerical approach might be needed. A good molecular model needs to produce the desired spectral density discussed above or to explain the cause of the specific powerlaw friction kernel. Further studies are needed to provide better physical insights into the nature of the slow motion and the understanding of the interesting power law nicely demonstrated in the single-molecule experiments of Xie and co-workers.

\section{ACKNOWLEDGMENTS}

J.T. acknowledges the support by the James W. Glanville Foundation at the California Institute of California while a major part of the work was completed. The authors thank R. A. Marcus for stimulating discussion. J.T. also thanks X. S. Xie, A. H. Zewail, H. Yang, M. K. Prakash, and M. E. Michel-Beyerle for discussions about the issues concerning the ps and ns fluorescence-decay components in flavoproteins. The help by B. Leigh and B. Tang in preparation of Figs. 1 and 2 is acknowledged. 
[1] W. E. Moerner, Rev. Sci. Instrum. 74, 3597 (2002); W. E. Moemer, Science 265, 46 (1994).

[2] Single Molecule Spectroscopy, edited by R. Rigler, M. Orrit, and T. Basché (Springer-Verlag, Heidelberg, 2002).

[3] S. M. Nie, D. T. Chiu, and R. N. Zare, Science 266, 1018 (1994); S. M. Nie and R. N. Zare, Science 26, 567 (1997).

[4] S. Weiss, Science 283, 1676 (1999); A. M. Kelley, X. Michalet, and S. Weiss, ibid. 292, 1671 (2001).

[5] H. P. Lu, L. Y. Xun, and X. S. Xie, Science 282, 1877 (1998).

[6] H. Yang, G. Luo, P. Karnchanaphanurach, T. M. Louie, I. Rech, S. Cova, L. Xun, and X. S. Xie, Science 302, 262 (2003).

[7] R. Zwanzig, Acc. Chem. Res. 23, 148 (1990).

[8] J. Wang and P. Wolynes, Chem. Phys. Lett. 212, 427 (1993); J. Wang and P. Wolynes, Chem. Phys. 180, 141 (1994).

[9] R. W. Pastor, R. Zwanzig, and A. Szabo, J. Chem. Phys. 105, 3878 (1996); D. J. Bicout, A. M. Berezhkovskii, and A. Szabo, ibid. 114, 2293 (2001).

[10] A. V. Barzykin, K. Seki, and M. Tachiya, J. Chem. Phys. 117, 1377 (2002).

[11] E. Barkai, R. Metzler, and J. Klafter, Phys. Rev. E 61, 132 (2000); R. Metzler, E. Barkai, and J. Klafter, Phys. Rev. Lett. 82, 3563 (1999).

[12] H. Yang and X. S. Xie, J. Chem. Phys. 117, 10965 (2002).

[13] S. C. Kou and X. S. Xie, Phys. Rev. Lett. 93, 180603 (2004).

[14] W. Min, G. Luo, B. J. Cherayil, S. C. Kou, and X. S. Xie, Phys. Rev. Lett. 94, 198302 (2005).

[15] H. Yang and X. S. Xie, Chem. Phys. 284, 423 (2002).

[16] R. Granek and J. Klafter, Phys. Rev. Lett. 95, 098106 (2005).

[17] P. Debnath, W. Min, X. S. Xie, and B. J. Cherayil, J. Chem. Phys. 123, 204903 (2005).

[18] J. Tang and R. A. Marcus, Phys. Rev. E 73, 022102 (2006).

[19] R. A. Marcus and N. Sutin, Biochim. Biophys. Acta 811, 265 (1985).

[20] J. B. Winkler and H. Gray, Proc. Natl. Acad. Sci. U.S.A. 102, 3534 (2005).

[21] C. C. Moser, J. M. Keske, K. Warncke, R. S. Farid, and P. L. Dutton, Nature (London) 355, 796 (1992).

[22] M. Whitlow, A. J. Howard, J. F. Wood, E. W. Voss, and K. D. Hardman, Protein Eng. 8, 749 (1995).

[23] P. E. Rouse, J. Chem. Phys. 21, 1272 (1953).

[24] R. Kimmich and N. Fatkullin, Adv. Polym. Sci. 170, 1 (2004).

[25] M. Doi and S. F. Edwards, The Theory of Polymer Dynamics (Clarendon Press, Oxford, 1986), Chap. 4.

[26] S. L. Yang, J. B. Witkoskie, and J. S. Cao, J. Chem. Phys. 117, 11010 (2002)

[27] M. P. Solf and T. A. Vilgis, Phys. Rev. E 55, 3037 (1997).
[28] A. Palazoglu, A. Gursoy, Y. Arkun, and B. Erman, J. Comput. Biol. 11, 1149 (2004).

[29] M. Y. Shen and K. F. Freed, J. Chem. Phys. 118, 5143 (2003); W. H. Tang, X. Y. Chang, and K. F. Freed, ibid. 103, 9492 (1995).

[30] G. La Penna, M. Mornino, F. Pioli, A. Perico, R. Fioravanti, J. M. Gruschus, and J. A. Ferretti, Biopolymers 49, 235 (1999).

[31] S. Ferree and H. W. Blanch, Biophys. J. 87, 468 (2004).

[32] G. Zaccai, Science 288, 1604 (2000).

[33] L. Corodone, M. Ferrand, E. Vitrano, and G. Zaccai, Biophys. J. 76, 1043 (1999).

[34] X. F. Zhou, J. L. Sun, H. J. An, Y. C. Buo, H. P. Fang, C. Su, X. D. Xiao, W. H. Huang, M. Q. Li, W. Q. Shen, and J. Hu, Phys. Rev. E 71, 062901 (2005).

[35] D. Cremer, A. Wu, A. Larsson, and E. Kraka, J. Mol. Model. 6, 396 (2000).

[36] A. Ansari, J. Chem. Phys. 110, 1774 (1999).

[37] A. Guillerno and J-P. C. Addad, J. Chem. Phys. 116, 3141 (2002).

[38] M. E. Ries, P. G. Klein, M. G. Brereton, and I. M. Ward, Macromolecules 31, 4950 (1998).

[39] G. Tsolou, V. G. Mavrantzas, and D. N. Theodorou, Macromolecules 38, 1478 (2005).

[40] A. Arbe, J. Colmenero, D. Richter, M. Monkenbusch, L. Willner, and B. Farago, Pramana, J. Phys. 63, 33 (2004).

[41] D. J. Plazek, E. Schlosser, A. Schönhals, and K. L. Ngai, J. Chem. Phys. 98, 6488 (1993).

[42] S. H. Lin, J. Chem. Phys. 44, 3759 (1966); J. Tang, Chem. Phys. Lett. 227, 170 (1994).

[43] K. Huang and A. Rhys, Proc. R. Soc. London, Ser. A 204, 406 (1950).

[44] J. Tang and R. A. Marcus, Phys. Rev. Lett. 95, 107401 (2005); J. Tang and R. A. Marcus, J. Chem. Phys. 123, 054704 (2005).

[45] J. Tang, S. N. Dikshit, and J. R. Norris, J. Chem. Phys. 103, 2873 (1995).

[46] W. C. Chen and J. Tang (private communication).

[47] D. Zhong and A. H. Zewail, Proc. Natl. Acad. Sci. U.S.A. 98, 11867 (2001).

[48] W. Min and X. S. Xie, Phys. Rev. E 73, 010902(R) (2006).

[49] V. B. P. Leite, L. C. P. Alonso, M. Newton, and J. Wang, Phys. Rev. Lett. 95, 118301 (2005).

[50] J. N. Ounchic and P. G. Wolynes, J. Chem. Phys. 98, 2218 (1993).

[51] G. R. Kneller and K. Hinsen, J. Chem. Phys. 121, 10278 (2004).

[52] G. R. Kneller, Phys. Chem. Chem. Phys. 7, 2641 (2005). 